Acta Cryst. (2002). A58 (Supplement), C5

X-RAY STRUCTURES OF THE UNIVERSAL TRANSLATION INITIATION FACTOR IF2/EIF5B: CONFORMATIONAL CHANGES ON GDP AND GTP BINDING

A. Roll-Mecak ${ }^{1}$ C Cao ${ }^{2}$ T.E. Dever $^{2}$ S.K. Burley ${ }^{1}$

1 The Rockefeller University 1230 York Avenue \#367 New York NY 10021 USA ${ }^{2}$ National Institutes of Health

X-ray structures of the universal translation initiation factor IF2/eIF5B have been determined in three states: free enzyme, inactive IF2/eIF5B-GDP, and active IF2/eIF5B-GTP. IF2/eIF5B is a conserved GTPase that facilitates ribosomal subunit joining and Met-tRNAi binding to ribosomes in all three kingdoms of life. The 'chalice-shaped' protein consists of an N-terminal G domain (I), plus and EF-Tu-type $\beta$-barrel (II) followed by a novel $\alpha / \beta / \alpha$ sandwich (III) connected via a long $\alpha$-helix to a second EF-Tu-type $\beta$-barrel (IV). Structural comparisons reveal a molecular lever, which amplifies a modest conformational change in the Switch 2 region of the $\mathrm{G}$ domain induced by $\mathrm{Mg}^{2+} / \mathrm{GTP}$ binding over a distance of $90 \AA$ from the $\mathrm{G}$ domain active center to domain IV. IF2/eIF5B interacts with eIF1 A through its C-terminal $\beta$-barrel domain. This region is critical for growth in vivo and translation in vitro. Interactions with the ribosome and other components of the translational apparatus are discussed. A model for the mechanism of action of IF2/eIF5B is proposed.

Keywords: TRANSLATION INITIATION GTPASE CONFORMATIONAL CHANGE
Acta Cryst. (2002). A58 (Supplement), C5

THE DISULFIDE BOND ISOMERASE DSBC IS SPECIFICALLY ACTIVATED BY THE IG FOLD DOMAIN OF THE ELECTRON TRANSPORTER DSBD

P.W. Haebel D. Goldstone P. Metcalf

Eth Zurich Institute For Molecular Biology And Biophysics Eth Hoenggerberg, Hpk Zurich Ch-8093 SWITZERLAND

Disulfide bonds are important for the structure and function of many proteins, including hormones, antibodies and bacterial toxins. The oxidative folding of proteins with disulfide bonds is catalyzed by thiol oxidoreductases in both bacteria and eukaryotes. The bacterial disulfide bond isomerisation pathway, consisting of DsbC and DsbD, rearranges incorrect disulfide bonds that trap proteins in non-functional conformations. The E. coli protein disulfide bond isomerase $\mathrm{DsbC}$ interacts with misfolded proteins and facilitates refolding by rearranging disulfide bonds. DsbC is a V-shaped homodimer with two catalytic domains facing one another across a central uncharged cleft that is the proposed binding site for substrates. The isomerase is specifically activated by the transmembrane electron transporter DsbD. The $\alpha$ domain of the inner membrane protein DsbD has an immunoglobulin fold with two active site cyteines that transport electrons. The intermediate of the electron transport reaction was trapped yielding a covalent DsbC-DsbDa complex. The $2.3 \AA$ crystal structure of the complex shows DsbD $\alpha$ binding into the central cleft of dimeric DsbC. The V-shaped DsbC molecule assumes a closed conformation on complex formation allowing both $\mathrm{DsbC}$ active sites to interact with $\mathrm{DsbD} \alpha$. These results provide the first insight into the electron transport process associated with oxidative protein folding and explain how DsbC is selectively activated by DsbD using electrons transported from the cytoplasm.

Keywords: PROTEIN DISULFIDE ISOMERASE, OXIDOREDUCTASES, ELECTRON TRANSPORT

Acta Cryst. (2002). A58 (Supplement), C5

\section{CRYSTAL STRUCTURE OF A BACTERIAL RNA POLYMERASE HOLOENZYME AT 2.6Å RESOLUTION}

D. G. Vassylyev ${ }^{1}$ S. Sekine ${ }^{1}$ O. Laptenko ${ }^{2}$ J. Lee $^{2}$ M.N. Vassylyeva ${ }^{3}$ S. Borukhov ${ }^{2}$ S Yokoyama ${ }^{1,3,4}$

${ }^{1}$ Cellular Signaling Laboratory and Structurome Research Group, RIKEN Harima Institute at spring-8, 1-1-1 Kouto, Mikazuki-cho, Sayo, Hyogo 6795148, Japan ${ }^{2}$ Department of Microbiology, SUNY Health Science Center, 450 Clarkson Avenue, Brooklyn, New York, 11203, USA ${ }^{3}$ RIKEN Genomic Sciences Center, 1-7-22 Suehiro-cho, Tsurumi, Yokohama 230-0045, Japan ${ }^{4}$ Department of Biophysics and Biochemistry, Graduate School of Science, University of Tokyo, 7-3-1 Hongo, Bunkyo-ku, Tokyo 113-0033,

In bacteria, the binding of a single protein, the initiation factor sigma, to a multi-subunit RNA polymerase core enzyme results in the formation of a holoenzyme, the active form of RNA polymerase essential for transcription initiation. We have determined the crystal structure of a bacterial RNA polymerase holoenzyme (the assembly of six protein subunits with a total molecular mass of about $450,000 \mathrm{KDa}$ ) from Thermus thermophilus at $2.6 \AA$ resolution $(\mathrm{R}$-factor $=22.8 \%$; $\mathrm{R}$-free $=27.4 \%)$. The holoenzyme structure provides insight into the structural organization of transcription intermediate complexes, and suggests implications for the mechanism of transcription initiation.

Keywords: RNA POLYMERASE HOLOENZYME, BACTERIA, STRUCTURE them together. Here each ligand is clamped between domains of one receptor molecule and dimerization occurs at a separate receptor:receptor interface. Thus a change in the conformation of the receptor upon ligand binding appears to be an important step in signal transmission into the cell. The structure of erbB-2 shows features of what an unligated receptor might look like and how this receptor can hetero-dimerise without binding ligand.

Keywords: CYTOKINE RECEPTORS COMPLEX CELL SIGNALLING 